\title{
Linx
}

Revue des linguistes de l'université Paris X Nanterre

$7 \mid 1995$

Saussure aujourd'hui

\section{La critique de la théorie saussurienne d'après Motoki Tokiéda (1941)}

Eisuke Komatsu

\section{OpenEdition}

1 Journals

Édition électronique

URL : http://journals.openedition.org/linx/1163

DOI : 10.4000/linx.1163

ISSN : 2118-9692

Éditeur

Presses universitaires de Paris Nanterre

\section{Édition imprimée}

Date de publication : 1 septembre 1995

Pagination : 258-262

ISSN : 0246-8743

\section{Référence électronique}

Eisuke Komatsu, « La critique de la théorie saussurienne d'après Motoki Tokiéda (1941) », Linx [En

ligne], 7 | 1995, mis en ligne le 13 juillet 2012, consulté le 10 décembre 2020. URL : http://

journals.openedition.org/linx/1163; DOI : https://doi.org/10.4000/linx.1163

Ce document a été généré automatiquement le 10 décembre 2020.

Département de Sciences du langage, Université Paris Ouest 


\title{
La critique de la théorie saussurienne d'après Motoki Tokiéda (1941)
}

\author{
Eisuke Komatsu
}

1 Dans le cadre de la réception de la théorie de Saussure, - puisque ce thème a été proposé par l'organisateur - je voudrais parler de la critique de la théorie saussurienne, car la critique est aussi une sorte de réception. Or la critique dont il s'agit date de l'année 1941, voici déjà un demi-siècle. Les remarques en sont souvent primitives, mais touchent de temps en temps à l'essentiel.

2 Je raconterai d'abord une anecdote : un jour Saussure s'entendit demander par un de ses étudiants: «qu'est-ce qu'un sujet parlant?» Sa réponse: «demandez à votre sens linguistique; impossible : trois unités. image : une unité. Tout le monde est-il d'accord? Alors, c'est la sanction sociale ${ }^{1}$.» La leçon de Saussure serait celle-ci : respectez votre intuition. N'ayez aucun critère de jugement préalable à l'observation. Il n'y a pas de méthode a priori. Ce qui est donné à votre sens constitue le seul critère de jugement. Dans ce sens, tout les membres de l'espèce humaine sont sur un pied d'égalité.

3 Je voudrais faire ici un bref résumé des réflexion de Tokiéda à ce sujet: $1^{\circ}$ la méthodologie construit dès l'abord l'objet de sa recherche. Or c'est cela même qui serait une faute grave. $2^{\circ} \mathrm{Si}$ toutes les théories linguistiques prétendent à l'universalité, y compris la théorie chomskienne, elles doivent expliquer les phénomènes très complexes d'une langue comme le japonais. Devant ces phénomènes extrêmement variés, vous vous apercevrez vite que l'idée de la langue comme unité identique à elle-même disparaît. Néanmoins, il ne me semble pas que Tokiéda voulait expulser toute méthodologie. La connaissance est acquise à travers notre expérience, par exemple dans I'acte du langage, mais sera travaillée dans un second temps par la méthode. On aperçoit dans les cours de Saussure deux niveaux de la description : l'un livré au niveau de l'observation, l'autre au niveau de la conscience jugeante. La théorie saussurienne n'est pas en fait une théorie, mais plutôt une réflexion sur le langage. On dit souvent que toutes les théories de la 
connaissance sont tributaires de la pensée de Husserl. On ne peut éviter la formalisation. On ne peut se dispenser de la discussion dans l'état idéal. Mais il faut dépasser de telle ou telle façon la méthode husserlienne. Tels sont les problèmes qui nous occupent dans cette décade « Saussure aujourd'hui. »

On sait bien que le fameux Cours de linguistique générale (nous le désignerons désormais par le sigle CLG) de Ferdinand de Saussure est une sorte de résumé des cours professés à l'Université de Genève pendant six ans. On conçoit aussi que le problème se pose de savoir si ce résumé incarne bien la pensée de Saussure. L'édition critique d'Engler nous montre abondamment tout ce qui est occulté derrière le livre reconstitué par deux disciples aussi fidèles aux idées de leur grand maître qu'à la pensée dominante de leur époque.

montré lors du congrès Saussure de Tokyo, que le personnage principal en était, outre Durkheim et les autres, Edmund Husserl, dont la pensée dominait tout le domaine de la science positiviste de cette époque. L'important ici, est de ne pas tomber dans une lecture réductrice et téléologique du texte final du CLG. C'est pourquoi je voudrais soulever ici le problème du sujet parlant.

6 Permettez-moi de vous présenter rapidement les reproches que Tokiéda adresse à Saussure ${ }^{2}$.

7 Au début du 19e siècle le monde occidental a vu naître la linguistique moderne. A partir de l'ère Méiji les Japonais n'ont pas échappé à ce grand courant. A la fin du 19e siècle, l'apparition de la linguistique synchronique a ouvert de nouveaux horizons, en opposition à la linguistique historique dite linguistique diachronique. Mais cette opposition ne fut un orage que dans le ciel occidental. La linguistique japonaise disposait d'une longue tradition propre, fondée sur une non moins longue tradition littéraire. Le Cours de linguistique générale de Saussure a été traduit en japonais en 1928 par Hidéo Kobayashi et a soulevé beaucoup de discussions touchant à l'idée et à la pensée de Saussure. Ce livre du grand maître n'est pas en effet transparent, mais difficile, et contradictoire de temps à autre. Tokiéda commence par analyser l'emploi que Saussure fait du mot « langue ». La linguistique commence par l'observation des faits linguistiques. Tokiéda s'accorde avec Saussure sur ce point. Mais ces faits eux-mêmes doivent être " concrets ", perçus à travers notre expérience, avant d'être réduits dans un second temps par la conscience jugeante à l'état de phénomène linguistique. «La langue est le processus des actes linguistiques. »dit-il.

8 Saussure au contraire voulait chercher un élément d'unité qui serait posé dès le début et identique à lui-même C'est la langue. « La langue », dit Saussure, " est un tout en soi et un principe de classification. » (CLG. p. 25).

9 Voici la traduction de la conclusion que Tokiéda apporte à ses propos dans son livre principal, Gengogaku genron (Principes de linguistique) publié en 1941.

«Saussure a voulu, pour des raisons méthodologiques, ne pas prendre comme objet le langage concret qui est notre expérience. Il a voulu isoler à l'intérieur du langage concret, hétérogène et multiforme, quelque chose qui soit homogène et uniforme. Il a fait de cette entité l'objet de sa recherche, l'a appelée "langue ", l'a reconnue comme étant un objet psychique, association d'une image acoustique et d'un concept, et l'a définie comme ayant une existence séparée, étant un fait social extérieur à l'individu. La langue prise comme objet est censée posséder une organisation structurée. Elle n'a de lien avec le sujet parlant que quand il l'utilise. Mais Saussure ne définit pas clairement le lien entre le sujet et cet objet ainsi utilisé. La plus grande contradiction de Saussure c'est que, si la langue est l'objet de la linguistique l'observation concrète ne peut se faire que sur la parole. Toute la 
théorie de Saussure n'est alors que le résultat d'une sorte d'objectivation du langage pour répondre à des préoccupations méthodologiques. » (Traduction par Catherine Garnier). Dès cette époque déjà, il a défini la méthode structuraliste et fait remarquer sa limite, qui consistait dans une description formelle et structurelle de son objet. En outre Saussure avait-il expulsé l'homme du phénomène humain par excellence, qu'est la linguistique. Enfin, toute enquête dialectale dans une région montagneuse de Suisse montrera vite que la langue s'y trouve infiniment divisée : l'idée de langue n'est donc qu'une conception idéale imposée par la science moderne. L'observation de Tokiéda nous incite à réexaminer la composition du texte du CLG.

11 On sait bien que le troisième cours constitue la base du CLG. Or le troisième cours commence par présenter les langues (Chap. 1 : diversité géographique de la langue). C'est seulement au second semestre que Saussure livre sa théorie générale de la langue. Bally et Sechehaye ont renversé et modifié la pensée de leur maître avec l'idée fondamentale de ce cours :

La linguistique a pour unique et véritable objet la langue envisagée en elle-même et pour elle-même. » (CLG. p. 317).

Cette expression n'est qu'un calque de Durkheim :

«La pensée collective (..) doit être étudiée en elle-même, pour elle-même (Les règles de la méthode sociologique, préface, 1901).

La comparaison avec le jeu d'échecs est commune aux Recherches logiques (1901) de Husserl, jusqu'à l'emploi des mots «Pièces en ivoire ou en bois ». Les influences que les penseurs et philosophes de la fin du 19e siècle et du début du 20e siècle -ont exercées sur Bally et Sechehaye ont fait du CLG un livre si idéologique qu'on a pu en tirer la conclusion rapide que c'était une Bible ou un livre fondateur du structuralisme. auteurs s'entendent pour affirmer que la langue n'existe pas en soi comme une "pierre » ou un « arbre » existe devant nous. « Le langage comme processus » que présente Tokiéda ressemble beaucoup au critère du jugement selon Saussure : « une unité ressentie par le sujet parlant ».

'opacité et les contradictions du CLG s'éclairciront un peu, si nous lisons celui-ci selon la perspective du cours tel qu'il a été donné à Genève. Un exemple: un chapitre de l'Appendice à l'Introduction - Principes de phonologie (p 63 du CLG) - semble curieusement déplacé dans un ouvrage consacré à la linguistique de la langue dont il ne présente que l'aspect articulatoire. Une explication convaincante nous sera donnée plus tard dans la première et deuxième partie du livre (Nature du signe liguistique. p. 97 et l'Aspect matériel de la valeur. p. 163). Nous retrouverons enfin ici l'aspect acoustique. Le premier appartenait au début du premier cours et les seconds sont tirés du deuxième et troisième cours. L'image acoustique dont parle le troisième cours n'est plus la voix naturelle. C'est déjà un sort réglé, réduire les espacements phénoménologiquement et articulé du point de vue linguistique. Tokiéda et Saussure me semblent dire que le monde idéal ne sera atteint qu'à travers notre expérience et c'est dans ce sens qu'ils disent que la langue est un acte.

La théorie que Tokiéda donne du sujet ne réussit pas cependant à expliquer complètement le japonais, dont le langage de politesse, par exemple, est particulièrement complexe et difficile à élucider d'autant plus qu'il ne sera plus réduit dans l'unité fonctionnelle de la langue. L'idée de sujet n'est pas identique chez les deux auteurs, ce qui 
soulèvera beaucoup de discussions, mais ce n'est pas ici le lieu de développer ces problèmes.

16 En dernier lieu, je voudrais vous présenter la situation actuelle des études saussuriennes au Japon. On sait que la première traduction du CLG a été publiée en 1928. Depuis, de nombreux savants, dont Tokiéda, n'ont cessé de s'intéresser aux analyses saussuriennes, mais la polémiques s'est toujours poursuivie fondée sur un caractère très discutable du CLG. Après le grand courant existentialiste, les idées du CLG ont attiré le regard de certains structuralistes. A l'heure actuelle avec le recul du structuralisme et l'émergence du post-structuralisme, nous lisons très attentivement le deuxième fascicule de l'édition Engler qui est constitué par des fragments expulsés lors de la rédaction du CLG. suivantes: 1) la Bibliothèque publique et universitaire de Genève 2) la Bibliothèque nationale de Paris 3) la Bibliothèque Houghton, à l'Université Harvard 4) les Archives scientifiques et académiques de Leningrad. Tous ces manuscrits ont été introduits au Japon, sauf ceux de Leningrad, sous forme de microfilms, publiées sur papier et mis à la disposition de tous. Ces manuscrits comptent près de 10.000 pages. Les Cahiers Ferdinand de Saussure (CFS), d'autre part, a publié dans son numéro 27 (1970 - 72) toute la correspondance échangée entre Saussure et Baudouin de Courtenay, à partir des manuscrits de la Bibliothèque deLeningrad. (1992)

\section{NOTES}

1. Cette anecdote est assez fictive. Mais Saussure parle souvent de la conscience du sujet parlant dont il faut partir quand il s'agit des phénomènes linguistiques. «La langue n'existe pas comme entité, mais seulement les sujets parlants. » (IIe cours, éd. E/ 98) note postérieure.

2. L'article de Catherine Garnier analyse bien le point d'opposition entre ces deux maîtres dans la revue Langages $n^{\circ} 68$ « La linguistique japonaise ». 